\title{
Renewable Natural Resources and Endogenous Growth*
}

\author{
J. Aznar-Márquez ${ }^{\dagger}$ J. R. Ruiz-Tamarit ${ }^{\ddagger \S}$
}

July, 2002.

\begin{abstract}
We study a two-sector endogenous growth model where a single consumption good is obtained using a renewable resource in combination with physical capital. Both inputs are essential for production and technical substitutes. In this context we analyze the issues of sustainability, long-run and short-run growth as well as convergence, associated with the competitive equilibrium solution trajectories. We show that efficiency, long-run growth and sustainability are both compatible in a natural resource based production economy.
\end{abstract}

Keywords: Natural Capital, Endogenous Growth, Sustainability, Convergence.

JEL classification: C62, D90, O41, Q20.

Running Head: Natural Resource and Growth.

*J. Aznar-Márquez acknowledges the support of the Belgian research programmes "Poles d'Attraction inter-universitaires" PAI P4/01, and "Action de Recherches Concertée" 99/04-235. J. R. Ruiz-Tamarit acknowledges financial support from the Spanish CICYT, Projects SEC99-0820 and SEC2000-0260.

†IRES, Université Catholique de Louvain (Belgium) and Universitat de València (Spain)

‡Universitat de València (Spain). Phone: (+) 3496 3828250. Fax: (+) 34963828249. E-mail: ramon.ruiz@uv.es

Corresponding author. J.R. Ruiz-Tamarit; Department of Economic Analysis; Avda. dels Tarongers s/n; E-46022 VALENCIA; SPAIN. 


\section{Introduction}

It is commonly accepted in growth literature that there is a strong connection between natural resource endowments and economic growth results. Evidence accumulated on historical examples and other ones taken from the post-world-war II period are used to take positions on this matter. As was early pointed out in Sachs and Warner (1995), countries with abundant natural resources tend to grow more slowly than countries poorly endowed in terms of any of the usual natural resources. A negative relationship between high natural resource intensity, namely a high value of resource-based exports to GDP, and the rate of growth appears as a robust empirical fact, with many examples showing resource-poor economies that outperform resourcerich economies in economic growth. In line with this result, Sachs and Warner (2001) even speak about the curse of natural resources after having explored, and refused statistically, the possibility of a spurious negative correlation arising from the fact that natural resource sector could be the only surviving sector in slow-growth countries.

There is, however, the traditional viewpoint according to which most of the current rich economies once developed with the aid of their natural resources. This argument seems to work as a counterexample only for periods, regions and natural resources for which high transportation costs made proximity decisive for physical availability. On the other hand, it is now well documented that such historical examples correspond to countries where the natural resource intensity, measured as a percent of GDP, was much smaller than the ratios observed in the examples corresponding to the second half of the twentieth century.

Across the literature we may find multiple social, political and economic factors explaining the adverse effects of resource abundance on growth. Nevertheless, the most accepted explanation of this focus on two elements: the direct effect known as Dutch disease (unfavorable exchange rate with high price levels and wages, as well as dependance on primary exports); and the indirect crowding-out effect that flows through human and social capital deterrence. Gylfason (2001a, 2001b) analyzes empirically these effects and finds that in most countries that are rich in natural resources, the rates of growth of per capita production over the long term have been lower than in other countries that are less well endowed. Hence, economic growth varies inversely with natural resource abundance. However, according to this author it has 
to be emphasized that the problem is not the existence of natural wealth as such, but rather the failure of public authorities to avert the dangers that accompany the gifts of nature. In any case, it is not inevitable that abundant natural resources prevent the emergence of a dynamic economy or that the discovery of such resources acts to dampen an already developed economy. Natural resources can be a blessing or a curse as long as they bring risks: abundant natural resources may imbue people with a false sense of security and lead governments to lose sight of the need for good economic management. Because of this, and given that countries without natural resources have a smaller margin for error, they are more likely to be efficiently managed in search of growth improvements. Auty (2001), in turn, considers two kind of development models on the basis of the attributes and qualities of political institutions: the competitive industrialization model that shows virtuous social and economic circles and applies to extreme resource-poor countries; and the staple trap model, which specially applies to resource-abundant countries. Rodríguez and Sachs (1999) propose an alternative explanation which consists in that resource-rich countries grow more slowly because they are living beyond their means. Hence, the corresponding adjustment for the level of per capita income to its steady state is made from above displaying negative rates of growth during the transition. These authors show that it can be optimal for resource-rich countries to overshoot their long-run equilibrium growth path.

Most of the literature concerning the relationship between natural resources availability and economic growth refers to non-renewable resources in the context of neoclassical exogenous growth models. In this paper, however, we come up with a two-sector endogenous growth model where a single consumption good is obtained using a renewable resource in combination with physical capital. Both inputs are assumed to be essential for production and, up to some degree, also technical substitutes. The renewable resource has the capacity to grow in size over time, being able to supply increasing productive inputs to the final good sector forever without bound. This capability, however, does not materialize automatically because it depends on the endogenously decided rate of extraction as well as on the exogenous intrinsic rate of growth. The latter results from demographic behavior of the natural resource stock and collective ecologically-based interventions. Therefore, we find in this point a first opportunity for public intervention through active ecological policies. Governmental organizations coordinated at different lev- 
els, mainly regional and local, can play a major role undertaking ecological and environmental actions that, while affecting the intrinsic rate of growth of natural resources, expand the margins for sustainability and economic growth.

Moreover, we assume that there is a private property rights system properly defined over the natural resource. Particularly, we assume that the stock of natural capital is equally and uniformly owned by consumers. Accordingly, firms have not open access to the stock because they have to pay the implicit unit price corresponding to the amount of harvested resource. On one hand, this allows us to avoid the problem of inefficient over-exploitation that usually appears in the context of commonly owned resources or in absence of property rights at all. On the other hand, we find here another opportunity for public interventions because governmental organizations, mainly national and international, have an important role to play by establishing a well-functioning legal system that enhances private property rights or wellmanaged common ownership.

In addition, we introduce a production externality in the final good sector associated with the natural resource. Although it is not fundamental for the results in our model, it enlarges the framework for discussion, and opens the possibility for multiple equilibrium paths in connection with the existence, at the aggregate level, of increasing returns to scale over accumulable factors. The presence of an externality in this model makes necessary to distinguish between the competitive equilibrium solution and the socially optimal solution. This allows again for a major role that government can play by means of a direct regulation. Optimal taxes and subsidies can be designed to create appropriate incentives for private behavior to eliminate the wedge between decentralized and social planner solutions.

Finally, we would like to point out, as Gylfason et al. (1999) did, that natural resource endowments may be a mixed blessing. Most people consider its abundance as a misfortune, but this is not necessarily so because after all positive, higher or lower, growth is in large measure a matter of choice, an endogenously determined outcome. What is important for economic growth is not the abundance of natural resources per se, but rather the efficiency of their management. Experience shows that abundant natural wealth, if not well managed, reduces economic growth in the long-run. It does provide nations with short term increased wealth but in the long term this may slow economic growth. For example, inasmuch as natural resource 
abundance involves free access to scarce common-property resources by private agents, leaving the resource rent up for grabs, inefficient harvesting and over-exploitation will necessarily appear. In this context, we will inquiry whether efficiency, long-run growth and sustainability are both compatible in a natural resource based production economy. In short, we are going to study the issues of sustainability, long-run and short-run growth as well as convergence, which appear associated with the competitive equilibrium solution trajectories.

The paper is organized as follows: in Section 2 we describe the model economy (preferences, technology and the characteristics of the natural renewable resource used in the production process); in Section 3 we solve the intertemporal optimization problem for a competitive economy, and provide the dynamic system which governs the state of the economy over time ${ }^{1}$; in Section 4 we present some Propositions collecting the main competitive equilibrium results for the relevant variables; in Section 5 we study economic features associated with equilibrium trajectories, particularly the properties of sustainability, growth and convergence; and finally, in Section 6 we conclude.

\section{The model}

We consider a closed and competitive economy which is populated by many identical and infinitely lived agents. Population, which we assume constant, is denoted by $N$. Individual preferences are assumed to be represented by an instantaneous utility function $U($.$) , which is twice continuously differentiable,$ strictly increasing and strictly concave in its argument $c(t)$, the real per capita consumption of an aggregate single good at date $t$. In particular, we assume the following functional form that implies a constant intertemporal elasticity of substitution, $\sigma^{-1} \lessgtr 1$, for consumption:

$$
U(c(t))=\frac{c(t)^{1-\sigma}-1}{1-\sigma}
$$

In this economy there is a renewable natural resource ${ }^{2}$, which is an essential

\footnotetext{
${ }^{1}$ The complete closed-form solution to this dynamic system is supplied in AznarMárquez and Ruiz-Tamarit (2002).

${ }^{2}$ According to the general definition given in Stiglitz (1980), we speak of a commodity or factor which is provided by nature and not produced, or producible, by man. Moreover, it is renewable in the sense of a stock that can be maintained, provided it is not consumed
} 
input for production and that is treated as a private good: it shares the properties of rivalry and excludability with the remaining inputs. The stock of this natural capital at date $t$ is denoted by $Q(t)$. We assume that such a stock is composed of homogeneous units and that it changes over time because of two different flows that have opposite and offsetting effects on the stock. First, in the absence of any human economically-based intervention, the natural resource evolves according to a biotic law of motion that suggests an exponential growth at a constant rate $\delta>0$. This implies that our natural resource is not subject to the traditional biological laws that apply to animal species, commonly represented by the logistic equation, or that we abstract from the negative feedbacks associated with overcrowding and environmental resistance. ${ }^{3}$ Beyond such phenomena, the intrinsic constant growth rate $\delta$ still may be considered as the net result of different exogenous natural processes: births, deaths and human ecologically-based interventions.

Second, the stock of natural capital is subject to an economically motivated extraction process, or harvesting activity, because it is necessarily required for production of the final good in our economy. We define $z(t)$ as the aggregate extraction rate, with $z(t) \in[0,1]$, and assume that there are many individual firms, each of them extracting a percentage $z_{i}(t)$ from the aggregate stock. If we consider every firm as being identical to each other, and assume that there are exactly the same number of firms than population, we get: $z(t)=\sum_{i=1}^{N} z_{i}(t)=N z_{i}(t)$. Finally, we assume a linear harvesting function according to which the renewable resource diminishes, each period, by the amount $z(t) Q(t)$. In short, we assume that resources used for harvesting are homogeneous, all the harvesters have the same objective function and the marginal product to effort is equal to the average one. Moreover, although the model considers free entry and costless harvesting ${ }^{4}$, there is no free disposal or open access to the natural capital stock because the individual firm has to pay for the use of the natural resource.

As we have just seen, the extraction rate is an endogenous variable, and the

too rapidly, or because its supply can be increased after utilization.

${ }^{3}$ It may be useful to see Peterson and Fisher (1977), as well as Shone (1997) and Perman et al. (1999), for an integrated study of all these biological and economic concepts from an analytical point of view.

${ }^{4}$ As Stiglitz (1980) points out, natural resouces require human activity to convert them into a useful form and indeed to extract them, but for simplicity we assume here that extraction costs are negligible. 
way the economy decides the value of $z(t)$ has direct effects on the opportunity set for present and future consumption. If the resource is harvested for too long at a rate exceeding its regeneration capabilities, the stock of natural capital will decrease over time. Consequently, the higher the current extraction rate the higher production and consumption today, but the smaller production and consumption in the near future. Connected with the previous statements, it must be recalled that even though the natural capital is a renewable resource, this does not mean that it should be inexhaustible. In fact, there is a crucial difference between physical and natural capital: while the first one may be used repeatedly without any consequence on its available quantity because only depreciation can reduce it, the second one disappears automatically from the stock as it is used for production. Now, combining the two flows that affect the evolution of this natural capital stock, we obtain the following law of motion: ${ }^{5}$

$$
\dot{Q}(t)=\delta(1-z(t)) Q(t)-z(t) Q(t)
$$

In the final single-good sector, production is ensured by many identical competitive firms. Technology at the firm level is represented by a production function $F($.$) , which is twice continuously differentiable in each of its argu-$ ments, strictly increasing and strictly concave in the private inputs, and satisfies the Inada conditions. Aggregate production, $Y(t)$, depends positively on the stock of homogeneous physical capital, $K(t)$, on the amount of natural resource that is harvested, destroyed or transformed each period, $z(t) Q(t)$, as well as on the aggregate stock of natural capital, $Q_{a}(t)$. The latter plays the role of an external effect in the production process because though all firms benefit from it, no individual harvesting decision can have a significant effect on the aggregate stock. Consequently, we will differentiate notation by adding the subscript $a$ to emphasize the distinction between internal and external effects. This externality affects productivity of all private factors of production and may be interpreted as the positive effect that the size of the natural stock exerts on the flow of produced goods, simply by augmenting total factor productivity or under the form of a lower implicit cost and a

\footnotetext{
${ }^{5}$ This expression may be encompassed in a more general law of motion for animal species that are subject to human harvesting: $\dot{Q}=\delta(1-z) Q\left(1-\frac{(1-z) Q}{S^{c}}\right)-z Q$. Our equation, then, comes immediately under the particular assumption of no saturation level or an infinite carrying capacity $S^{c}$, the maximum stock that the habitat can support.
} 
greater implicit quality associated with the harvested natural resource. ${ }^{6}$ The production function $F($.$) is assumed Cobb-Douglas: { }^{7}$

$$
Y(t)=A K^{\beta}(t)(z(t) Q(t))^{1-\beta} Q_{a}(t)^{\gamma}
$$

This equation implies that production for each firm exhibits constant returns to scale over private internal factors. Moreover, for fixed $Q_{a}$, there are diminishing returns to $K$ and $Q$. If, however, $Q_{a}$ rises along with $K$ diminishing returns will not arise because of the increasing returns to scale with respect to all the accumulable factors taken together. That is, there are increasing returns to scale at the aggregate and social levels. Note that the form of the production function implies that an increase in $Q_{a}$ raises the marginal products of both physical and natural capital, and the absence of diminishing returns to the factors that can be accumulated is at the origin of the endogenous growth result. ${ }^{8}$ On the other hand, in this equation the efficiency parameter $A$ roughly represents the constant technological level in the final good sector, parameter $\beta$ is the elasticity of output with respect to physical capital, and parameter $\gamma$ is a positive constant capturing the weight of the external effects of natural capital.

Total output, $Y(t)$, may be allocated to either aggregate consumption or physical capital accumulation. ${ }^{9}$ Consumption contributes directly to current welfare while investment, which increases current physical capital stock, allows for a greater future consumption and welfare. For the sake of simplicity, it is assumed that there is no physical capital depreciation. Hence, the current aggregate resources constraint may be written as:

\footnotetext{
${ }^{6}$ For example, in the case of the forest resource and wooded land a lower stock might imply either more time and a greater distance to find wood of a certain quality, which must be considered as a higher implicit price, or a lower quality of the harvested wood for a given price.

${ }^{7} \mathrm{We}$ abstract from labor as an explicit factor of production because it is assumed inelastically supplied. Each agent is endowed with a fixed quantity of work effort, which we normalize to one. Consequently, it may be considered implicitly included in the constant term of the production function.

${ }^{8}$ Under these assumptions, individual firms still face to a concave optimization problem, but the external effect introduces a distortion between private and social marginal productivity of natural capital.

${ }^{9}$ As previously set, we do not consider in this model explicit extraction costs, which are usually modelled as reducing the amount of output available for consumption and investment.
} 


$$
\dot{K}(t)=A K^{\beta}(t)(z(t) Q(t))^{1-\beta} Q_{a}(t)^{\gamma}-N c(t)
$$

\section{Solving the model}

The problem facing up this economy, for a given constant intertemporal discount rate $\rho>0$ that represents the time preference or the agents impatience degree, consists in choosing the controls $c(t)$ and $z(t) \forall t \geq 0$, which solve the following optimization problem:

$$
\operatorname{Max} \int_{0}^{\infty} \frac{c(t)^{1-\sigma}-1}{1-\sigma} N e^{-\rho t} d t
$$

subject to (2) and (4), for $K(0)=K_{0}>0$ and $Q(0)=Q_{0}>0$ given.

Because of the presence of a technological externality, our economy does not fulfil the required assumptions for the basic welfare theorems to be satisfied. Consequently, the competitive solution to this problem does not correspond to a social optimum. Namely, the intertemporal growth paths are not optimal growth paths in the sense of Pareto. In this paper we focus on the study of the competitive equilibrium and its outcomes, which is still compatible with the presence of increasing returns to scale because of the non-increasing private returns to both capitals. Therefore, the representative optimizing agent takes $Q_{a}(t)$ as given when he has to decide the variables under his direct control. In order to calculate the competitive sub-optimal equilibrium, we will impose hereafter an additional condition to make individual decisions compatible at the aggregate level. From now on, we will consider the constant population $N$ normalized to one and remove the time subscripts from the variables. The current value Hamiltonian associated with the previous intertemporal optimization problem may be written as:

$$
\begin{gathered}
H^{c}\left(K, Q, \theta_{1}, \theta_{2}, c, z ; \sigma, A, \beta, \gamma, \delta,\left\{Q_{a}(t): t \geq 0\right\}\right)= \\
=\frac{c^{1-\sigma}-1}{1-\sigma}+\theta_{1}\left[A K^{\beta}(z Q)^{1-\beta} Q_{a}^{\gamma}-c\right]+\theta_{2}[(\delta-(1+\delta) z) Q]
\end{gathered}
$$


where $\theta_{1}$ and $\theta_{2}$ are the co-state variables (shadow prices) associated with $K$ and $Q$, respectively. Then, the set of equations arising from the Pontryagin's Maximum Principle as first order necessary conditions, under the equilibrium condition $Q_{a}=Q$, are: ${ }^{10}$

$$
\begin{gathered}
c^{-\sigma}=\theta_{1} \\
\theta_{1}(1-\beta) A K^{\beta} z^{-\beta} Q^{1-\beta+\gamma}=\theta_{2}(1+\delta) Q \\
\dot{\theta}_{1}=\rho \theta_{1}-\theta_{1} \beta A K^{\beta-1} z^{1-\beta} Q^{1-\beta+\gamma} \\
\dot{\theta}_{2}=\rho \theta_{2}-\theta_{1}(1-\beta) A K^{\beta} z^{1-\beta} Q^{-\beta+\gamma}-\theta_{2} \delta\left(1-\left(\frac{1+\delta}{\delta}\right) z\right) \\
\dot{K}=A K^{\beta} z^{1-\beta} Q^{1-\beta+\gamma}-c \\
\dot{Q}=\delta\left(1-\left(\frac{1+\delta}{\delta}\right) z\right) Q
\end{gathered}
$$

The boundary conditions include the initial conditions $K_{0}$ and $Q_{0}$, and the transversality conditions:

\footnotetext{
${ }^{10}$ These equations correspond to the competitive solution of the decentralized optimization problem where each competitive firm faces the following stationary problem in the absence of extraction costs and depreciation charges: $\underset{\left\{K_{i}, z_{i}\right\}}{\operatorname{Max}} \pi_{i}=F\left(K_{i}, z_{i} Q, Q_{a}\right)-r K_{i}-$ $q z_{i} Q$, and the consumers side solves the intertemporal optimization problem (P) but taking into account the dynamic constraint $\stackrel{\dot{K}}{=}=r K+q z Q+\Pi-N c$ instead of (4). With price $q$ we signify that the natural resource is scarce, consequently explicit extraction costs are not needed to have a well-defined problem. The rental prices for both types of capital services in the second problem satisfy the necessary conditions from the former:

$$
r=F_{K}\left(K_{i}, z_{i} Q, Q_{a}\right)=\beta A K_{i}^{\beta-1}\left(z_{i} Q\right)^{1-\beta} Q_{a}^{\gamma}
$$$$
q=F_{2}\left(K_{i}, z_{i} Q, Q_{a}\right)=(1-\beta) A K_{i}^{\beta}\left(z_{i} Q\right)^{-\beta} Q_{a}^{\gamma} .
$$

Given the assumption of constant returns to scale at the private level, output exactly exhausts by paying the inputs according to their marginal productivities. Hence, aggregate economic rents $\Pi$ become zero.
} 


$$
\begin{aligned}
& \lim _{t \rightarrow \infty} \theta_{1} K \exp \{-\rho t\}=0 \\
& \lim _{t \rightarrow \infty} \theta_{2} Q \exp \{-\rho t\}=0
\end{aligned}
$$

Equation (6) shows that on the margin, final good must be equally valuable in its two uses: consumption and physical capital accumulation. Namely, the marginal utility of consumption must be equal to the marginal value (implicit price) of physical capital. Equation (7) shows in turn that, at equilibrium, the value of the marginal productivity of natural resource (in case of being harvested) must be equal to the value of its marginal contribution to natural capital accumulation (in case of being saved). The Euler equation (8) states that the marginal productivity of physical capital (the benefit of delaying consumption) equals its rental price, which in the absence of depreciation is given by the difference between the discount rate and the rate of physical capital gains or losses, $\rho-\frac{\dot{\theta}_{1}}{\theta_{1}}$. The Euler equation (9), in turn, gives the intertemporal efficiency condition that must be satisfied in the process of natural capital allocation. The value, in terms of natural capital, of the marginal productivity of $Q$ in the final good sector must be equal to its opportunity cost: the difference between the discount rate and the rate of change of the aggregate shadow value of the natural capital stock. This is a modified version of the Hotelling rule, which imposes intertemporal efficiency to the resource extraction activities. In our particular case, after substituting the static efficiency condition (7) into (9), we find that dynamic efficiency requires the intrinsic exogenous rate of growth of natural resource (the benefit of waiting) to be equal to the difference between the discount rate and the rate of capital gains or losses associated with natural capital (the opportunity cost), $\delta=\rho-\frac{\dot{\theta}_{2}}{\theta_{2}} \cdot{ }^{11}$

\footnotetext{
competitive rental prices may be written as:

$$
r=\rho-\frac{\dot{\theta}_{1}}{\theta_{1}}
$$$$
q=\frac{\theta_{2}}{\theta_{1}}\left(\rho+1-\frac{\dot{\theta}_{2}}{\theta_{2}}\right)=\frac{\theta_{2}}{\theta_{1}}(1+\delta)
$$

${ }^{11}$ Arising from the decentralized problem, under the equilibrium conditions we find that 
From (6) and (7) we get the next two control functions:

$$
\begin{gathered}
c=\theta_{1}^{-\frac{1}{\sigma}} \\
z=\left(\frac{(1-\beta) A}{(1+\delta)}\right)^{\frac{1}{\beta}}\left(\frac{\theta_{1}}{\theta_{2}}\right)^{\frac{1}{\beta}} Q^{\frac{\gamma}{\beta}-1} K
\end{gathered}
$$

After substituting the above expressions in (8)-(11), we obtain the following dynamic system:

$$
\begin{gathered}
\dot{\theta}_{1}=\rho \theta_{1}-\xi \theta_{1}^{\frac{1}{\beta}} \theta_{2}^{-\left(\frac{1-\beta}{\beta}\right)} Q^{\frac{\gamma}{\beta}} \\
\dot{\theta}_{2}=-(\delta-\rho) \theta_{2} \\
\dot{K}=\frac{\xi}{\beta} \theta_{1}^{\frac{1-\beta}{\beta}} \theta_{2}^{-\left(\frac{1-\beta}{\beta}\right)} K Q^{\frac{\gamma}{\beta}}-\theta_{1}^{-\frac{1}{\sigma}} \\
\dot{Q}=\delta Q-\left(\frac{1-\beta}{\beta}\right) \xi \theta_{1}^{\frac{1}{\beta}} \theta_{2}^{-\frac{1}{\beta}} K Q^{\frac{\gamma}{\beta}}
\end{gathered}
$$

where $K$ and $Q$ represent either the aggregate as well as the per capita levels of physical capital and natural capital, respectively, and $\xi \equiv \frac{\beta(1+\delta)}{(1-\beta)}\left(\frac{(1-\beta) A}{(1+\delta)}\right)^{\frac{1}{\beta}}$. These equations, together with the initial conditions $K_{0}$ and $Q_{0}$, and the transversality conditions (12) and (13), make the equilibrium dynamics of our economic system completely determined over time.

\section{Competitive equilibrium results}

The complete closed-form solution for the variables appearing in the dynamic system (16)-(19), as well as for the controls of the model, may be found in Aznar-Márquez and Ruiz-Tamarit (2002) together with a detailed proof of each result. This dynamic system, which mirrors the structure of the Lucas (1988) two-sector endogenous growth model, has been solved under the

These two prices share the usual structure for rental prices, adapted for the particular assumptions made in this model where the physical capital depreciation rate is zero, the flow of harvested natural capital authomatically implies a withdraw from the stock and prices are measured in terms of consumption good. 
assumption that the inverse of the intertemporal elasticity of substitution equals the physical capital share, $\sigma=\beta$. In a recent paper, Ruiz-Tamarit (2002) shows that the dynamic characterization of the solution must be differentiated according to the parameter subspace in which we undertake the study of the system. There are two disjoint sets of parameter values for which it does exist at least one solution trajectory under competitive conditions. The first one always implies a multiplicity of solutions and, hence, indeterminacy. It refers to the case of multiple solution trajectories starting from the same initial conditions for predetermined variables, all of them converging to a unique steady state or balanced growth path. Nevertheless, as it is shown there, this case is very implausible because of its counterintuitive predictions. For example, the long-run rate of growth emerges as positively related to the discount rate, while the rate of extraction from the natural resource stock shows a negative dependence on this parameter. Consequently, in this paper we concentrate on the parameter subspace where the results ensure the existence of a unique solution trajectory for every variable. Namely, the case where the externality is not too strong. Now, in this section we will collect the main results for the variables: natural capital stock, extraction rate, output, relative shadow prices and the ratio between the two capital stocks.

Proposition 1 : Under the equilibrium conditions, if $\gamma<\beta$ and $\delta(1+\gamma-\beta)-$ $\rho<0$ then:

(i) there exist a unique and positive equilibrium path for the renewable natural capital stock $Q$, starting from $Q_{0}$ :

$$
Q=Q_{0} \exp \left\{\frac{\delta-\rho}{\beta-\gamma} t\right\}
$$

(ii) there are no transitional dynamics for $Q$, which grows permanently along a balanced growth path at a positive or negative constant rate $\bar{g}_{Q}=\frac{\delta-\rho}{\beta-\gamma}$, depending on whether $\delta \gtrless \rho$.

In the complementary parameter subspace it does not exist any equilibrium path for $Q$ or there are a continuum of such equilibrium paths starting from $Q_{0}$.

Proof: See Aznar-Márquez and Ruiz-Tamarit (2002). 
Proposition 2 : Under the equilibrium conditions, if $\gamma<\beta$ and $\delta(1+\gamma-\beta)-$ $\rho<0$ then:

(i) there exist a unique equilibrium path for the extraction or harvesting rate $z$;

(ii) this equilibrium path, along which there are no transitional dynamics, may be characterized by the constant initial value $z(0)=\bar{z}=-\frac{\delta(1+\gamma-\beta)-\rho}{(1+\delta)(\beta-\gamma)}$, which also represents the unique balanced growth path;

(iii) the extraction rate satisfies the constraint $1>\bar{z}>0$ if and only if $\delta>\rho+\gamma-\beta$.

In the complementary parameter subspace it does not exist any equilibrium path for $z$ or there are a continuum of such equilibrium paths.

Proof: See Aznar-Márquez and Ruiz-Tamarit (2002).

Proposition 3 : Under the equilibrium conditions, if $\gamma<\beta$ and $-\left(\frac{\beta-\gamma}{\beta}\right) \rho<$ $\delta(1+\gamma-\beta)-\rho<0$ then:

(i) there exist a unique and positive equilibrium path for the physical capital stock $K$, starting from $K_{0}$ :

$$
\begin{aligned}
K= & \frac{\beta}{\rho}\left[\left\{\left(\frac{\rho}{\beta} K_{0}\right)^{1-\beta}-\frac{(\beta-\gamma) \beta C_{0}^{0} Q_{0}^{1+\gamma-\beta}}{\delta \beta(1+\gamma-\beta)-\gamma \rho}\right\} \exp \left\{-\frac{(1-\beta) \rho}{\beta} t\right\}\right. \\
& \left.+\frac{(\beta-\gamma) \beta C_{0}^{0} Q_{0}^{1+\gamma-\beta}}{\delta \beta(1+\gamma-\beta)-\gamma \rho} \exp \left\{\frac{(\delta-\rho)(1+\gamma-\beta)}{(\beta-\gamma)} t\right\}\right]^{\frac{1}{1-\beta}},
\end{aligned}
$$

where the constant $C_{0}^{0}=\left(\frac{1-\beta}{\beta}\right) \xi\left(\frac{\left(\frac{\gamma-\beta}{\beta}\right)(1-\beta) \xi \frac{\sigma}{\rho}}{\delta(1+\gamma-\beta)-\rho}\right)^{\beta-1}$ is positive;

(ii) this equilibrium path evolves describing transitional dynamics, and approaches asymptotically to the unique positive balanced growth path where the physical capital stock grows permanently, at a positive or negative constant rate $\bar{g}_{K}=\frac{1+\gamma-\beta}{1-\beta}\left(\frac{\delta-\rho}{\beta-\gamma}\right)$, depending on whether $\delta \gtrless \rho$. That is:

$$
\bar{K}=\frac{\beta}{\rho}\left(\frac{(\beta-\gamma) \beta C_{0}^{0} Q_{0}^{1+\gamma-\beta}}{\delta \beta(1+\gamma-\beta)-\gamma \rho}\right)^{\frac{1}{1-\beta}} \exp \left\{\frac{1+\gamma-\beta}{1-\beta}\left(\frac{\delta-\rho}{\beta-\gamma}\right) t\right\} .
$$

In the complementary parameter subspace it does not exist any equilibrium path for $K$ or there are a continuum of such equilibrium paths starting from $K_{0}$. 
Proof: See Aznar-Márquez and Ruiz-Tamarit (2002).

Proposition 4 : Under the equilibrium conditions $c=\frac{\rho}{\beta} K, \forall t \geq 0$.

Proof: See Aznar-Márquez and Ruiz-Tamarit (2002).

From the previous Propositions, it is easy to deduce the following one which summarizes in terms of the aggregate level of output, or the equivalent per capita level, the growth results in our economy.

Proposition 5 : Under the equilibrium conditions, if $\gamma<\beta$ and $-\left(\frac{\beta-\gamma}{\beta}\right) \rho<$ $\delta(1+\gamma-\beta)-\rho<0$ then:

(i) there exist a unique and positive equilibrium path for the per capita production $Y$, starting from $Y_{0}=A K_{0}^{\beta} Q_{0}^{1+\gamma-\beta}\left(-\frac{\delta(1+\gamma-\beta)-\rho}{(1+\delta)(\beta-\gamma)}\right)^{1-\beta}$ :

$$
\begin{gathered}
Y=A K^{\beta} z^{1-\beta} Q^{1+\gamma-\beta}=A\left(\frac{\beta}{\rho}\right)^{\beta} Q_{0}^{1+\gamma-\beta}\left(-\frac{\delta(1+\gamma-\beta)-\rho}{(1+\delta)(\beta-\gamma)}\right)^{1-\beta} \\
\cdot\left[\left(\frac{\rho}{\beta} K_{0}\right)^{1-\beta}-\frac{(\beta-\gamma) \beta C_{0}^{0} Q_{0}^{1+\gamma-\beta}}{\delta \beta(1+\gamma-\beta)-\gamma \rho}\right] \exp \left\{\frac{(1-\beta)[\delta(1+\gamma-\beta)-\rho]}{(\beta-\gamma) \beta} t\right\} \\
\left.+\frac{(\beta-\gamma) \beta C_{0}^{0} Q_{0}^{1+\gamma-\beta}}{\delta \beta(1+\gamma-\beta)-\gamma \rho} \exp \left\{\frac{(1+\gamma-\beta)}{\beta}\left(\frac{\delta-\rho}{\beta-\gamma}\right) t\right\}\right]^{\frac{\beta}{1-\beta}} ;
\end{gathered}
$$

(ii) this equilibrium path shows transitional dynamics, and approaches asymptotically to an exponential monotonic path along which production grows permanently at a positive or negative constant rate $\bar{g}_{Y}=\frac{1+\gamma-\beta}{1-\beta}\left(\frac{\delta-\rho}{\beta-\gamma}\right)$, depending on whether $\delta \gtrless \rho$. This one constitutes the unique positive balanced growth path. That is:

$$
\bar{Y}=\frac{A\left(\frac{\beta}{\rho}\right)^{\beta} Q_{0}^{\frac{1+\gamma-\beta}{1-\beta}}\left(-\frac{\delta(1+\gamma-\beta)-\rho}{(1+\delta)(\beta-\gamma)}\right)^{1-\beta}}{\left(\frac{\delta \beta(1+\gamma-\beta)-\gamma \rho}{(\beta-\gamma) \beta C_{0}^{0}}\right)^{\frac{\beta}{1-\beta}}} \exp \left\{\frac{1+\gamma-\beta}{1-\beta}\left(\frac{\delta-\rho}{\beta-\gamma}\right) t\right\} .
$$

In the complementary parameter subspace it does not exist any equilibrium path for $Y$ or there are a continuum of such equilibrium paths starting from $Y_{0}$. 
The next Proposition gives some additional results arising from the model, which concern the relative shadow prices and the ratio between the two capital stocks.

Proposition 6 : Under the equilibrium conditions, if $\gamma<\beta$ and $-\left(\frac{\beta-\gamma}{\beta}\right) \rho<$ $\delta(1+\gamma-\beta)-\rho<0$ then:

(i) there exist a unique and positive equilibrium path for the relative price $\frac{\theta_{1}}{\theta_{2}}$ and a unique and positive equilibrium path for the ratio $\frac{K}{Q}$, starting, respectively, from $\frac{\theta_{1}(0)}{\theta_{2}(0)}=Q_{0}^{\beta-\gamma}\left(\frac{\delta(1+\gamma-\beta)-\rho}{K_{0}\left(\frac{\gamma-\beta}{\beta}\right)(1-\beta) \xi}\right)^{\beta}>0$ and $\frac{K_{0}}{Q_{0}}$ :

$$
\begin{gathered}
\frac{\theta_{1}}{\theta_{2}}=\frac{Q_{0}^{\beta-\gamma}}{\left(\frac{\left(\frac{\gamma-\beta}{\beta}\right)(1-\beta) \xi \frac{\sigma}{\rho}}{\delta(1+\gamma-\beta)-\rho}\right)^{\beta}}\left[\left(\frac{\rho}{\beta} K_{0}\right)^{1-\beta}-\frac{(\beta-\gamma) \beta C_{0}^{0} Q_{0}^{1+\gamma-\beta}}{\delta \beta(1+\gamma-\beta)-\gamma \rho}\right] \exp \left\{-\frac{\delta(1-\beta)}{\beta} t\right\} \\
\left.+\frac{(\beta-\gamma) \beta C_{0}^{0} Q_{0}^{1+\gamma-\beta}}{\delta \beta(1+\gamma-\beta)-\gamma \rho} \exp \left\{\frac{\gamma}{\beta}\left(\frac{\delta-\rho}{\beta-\gamma}\right) t\right\}\right]^{\frac{-\beta}{1-\beta}} \\
\frac{K}{Q}=\frac{\beta}{\rho Q_{0}}\left[\left(\frac{\rho}{\beta} K_{0}\right)^{1-\beta}-\frac{(\beta-\gamma) \beta C_{0}^{0} Q_{0}^{1+\gamma-\beta}}{\delta \beta(1+\gamma-\beta)-\gamma \rho}\right] \exp \left\{-\frac{(1-\beta)(\delta \beta-\gamma \rho)}{\beta(\beta-\gamma)} t\right\} \\
\left.+\frac{(\beta-\gamma) \beta C_{0}^{0} Q_{0}^{1+\gamma-\beta}}{\delta \beta(1+\gamma-\beta)-\gamma \rho} \exp \left\{\gamma\left(\frac{\delta-\rho}{\beta-\gamma}\right) t\right\}\right]^{\frac{1}{1-\beta}}
\end{gathered}
$$

(ii) under the additional condition: $\delta \beta>\gamma \rho$, these equilibrium paths move asymptotically, describing transitional dynamics, towards their respective unique and positive balanced paths. Along the balanced path, relative price monotonically approaches to zero or grows exponentially to infinity at a constant rate, depending on whether $\delta \gtrless \rho$. On the contrary, along its balanced path, the ratio between capitals grows exponentially at a constant rate or decreases monotonically to zero, depending on whether $\delta \gtrless \rho$. That is:

$$
\left(\frac{\overline{\theta_{1}}}{\theta_{2}}\right)=\frac{\left(\frac{\delta \beta(1+\gamma-\beta)-\gamma \rho}{(\beta-\gamma) \beta C_{0}^{0}}\right)^{\frac{\beta}{1-\beta}}}{Q_{0}^{\frac{\gamma}{1-\beta}}\left(\frac{\left(\frac{\gamma-\beta}{\beta}\right)(1-\beta) \xi \frac{\sigma}{\rho}}{\delta(1+\gamma-\beta)-\rho}\right)^{\beta}} \exp \left\{\frac{-\gamma}{1-\beta}\left(\frac{\delta-\rho}{\beta-\gamma}\right) t\right\},
$$




$$
\left(\frac{\bar{K}}{Q}\right)=\frac{\beta}{\rho} Q_{0}^{\frac{\gamma}{1-\beta}}\left(\frac{(\beta-\gamma) \beta C_{0}^{0}}{\delta \beta(1+\gamma-\beta)-\gamma \rho}\right)^{\frac{1}{1-\beta}} \exp \left\{\frac{\gamma}{1-\beta}\left(\frac{\delta-\rho}{\beta-\gamma}\right) t\right\}
$$

(iii) in the particular case where no externality does exist, $\gamma=0$, the relative price $\frac{\theta_{1}}{\theta_{2}}$ follows a unique and positive equilibrium path approaching monotonically to the positive constant value: $\left(\frac{\delta}{\beta}\right)^{\frac{\beta}{1-\beta}} \frac{A(1-\beta)^{\frac{1}{1-\beta}}}{(1+\delta)^{1-\beta}}$, and the ratio $\frac{K}{Q}$ follows a unique and positive equilibrium path approaching monotonically to the positive constant value: $\left(\frac{\xi}{\delta}\right)^{\frac{1}{1-\beta}}\left(-\frac{\delta(1-\beta)-\rho}{(1-\beta) \xi}\right)$.

In the complementary parameter subspace it does not exist any equilibrium path for $\frac{\theta_{1}}{\theta_{2}}$ and $\frac{K}{Q}$ or there are a continuum of such equilibrium paths for each one.

The proof of this Proposition is easy from the previous results on $Q$ and $K$, as well as from the results in Aznar-Márquez and Ruiz-Tamarit (2002) relative to $\theta_{1}$ and $\theta_{2}$. In short, we have that when capital stocks increase (decrease), with $K$ increasing (decreasing) more rapidly than $Q$, their shadow prices decrease (increase), with $\theta_{1}$ going down (up) faster than $\theta_{2}$.

\section{Growth, Convergence and Sustainability}

In this section we interpret the previous analytical results stressing on the properties of the model in terms of long-run sustainability and growth as well as short-run growth and convergence. First, from Proposition 1 we have that $\bar{g}_{Q}$ is positive when $\delta>\rho$. In such a case, using the equilibrium value of the harvesting rate $\bar{z}$ provided by Proposition 2 , the result of permanent positive growth for $Q$ appears associated with a harvesting rate lower than $z^{S}=\frac{\delta}{1+\delta} \cdot{ }^{12}$ This condition also ensures a positive value for the long-run growth rates $\bar{g}_{K}$, $\bar{g}_{c}$ and $\bar{g}_{Y}$. In the alternative case where $\delta<\rho$, all the previous results are reversed and they come associated with a harvesting rate greater than $z^{S}$. In short, long-run positive growth is sustainable as long as $\bar{z}<z^{S}$. Given that $z^{S}$ is an increasing function of the intrinsic growth rate of the natural

\footnotetext{
${ }^{12}$ The value $z^{S}$ represents the harvesting rate for which the stock of natural capital (a renewable natural resource in this model) remains constant over time.
} 
capital, $\delta$, several exogenous factors affecting birth and death rates as well as off-the-model human ecologically-based interventions may have an important positive impact on the margins for sustainable long-run endogenous positive growth. Usually, by sustainability it is meant a state with a sustained longrun level of per capita consumption in which the natural capital stock, or its flow of resource services, is non-declining through time. In this paper we go further supplying conditions for a sustained long-run positive rate of growth of per capita consumption. Our results show that it is possible to have simultaneously economic growth and sustainability and, moreover, how are they compatible with efficient behavior in both senses: intra and intertemporally.

Second, the long-run value of the rate of growth associated with physical capital, consumption and (per capita) production, $\bar{g}$, depends positively on the efficiency parameter $\delta$, which represents the biotic rate of growth of natural capital, but negatively on the rate of discount, $\rho$. These two relations hold irrespective to the sign of growth: positive when $\delta>\rho$ and negative when $\delta<\rho$. On other hand, this rate of growth depends positively on the parameter associated with the technological externality, $\gamma$, when the economy experiences positive growth, $\delta>\rho$, but it changes to the opposite when there is negative growth, $\delta<\rho$. Finally, because of the presence of the externality, the effect of the physical capital share in goods sector, $\beta$, over the long-run rate of growth is ambiguous. However, as long as the value of $\gamma$ is small enough, we can expect a negative effect when $\delta>\rho$ and a positive one when $\delta<\rho$. The long-run, as well as the short-run, rate of growth of natural capital stock, $\bar{g}_{Q}$, depends positively on the parameter $\delta$ and negatively on $\rho$, regardless of the sign of growth. This rate, instead, depends negatively on $\beta$ and positively on $\gamma$ when the economy experiences positive growth, but it changes to the opposite when there is negative growth.

Moreover, looking at the long-run as well as the short-run equilibrium harvesting rate $\bar{z}$, we can see that it depends negatively on the intrinsic growth rate of the natural capital, $\delta$, and positively on the rate of discount, $\rho$, irrespectively to what happens in terms of growth. However, the harvesting rate increases with $\beta$ and decreases with $\gamma$ only when the rate of growth in the economy is positive, because these effects are reversed when the rate of growth is negative.

Consequently, an increase in $\delta$ that expands the margins for sustainability 
because increases $z^{S}$ and reduces $\bar{z}$, has an additional positive effect increasing the values of the long-run rates of growth $\bar{g}_{Q}$ and $\bar{g}_{Y}$. On the other hand, a more patient society chooses a lower harvesting rate and reaches a greater long-run rate of growth. The same is true for an economy with a stronger externality and a lower physical capital share, as long as such economy experiences positive growth. Overall these questions we have that in a more patient society, positive long-run growth is compatible with lower biotic rates of growth of its natural capital, while in less patient societies long-run positive growth is only possible under higher values for $\delta$.

In the short-run, however, from (20) the rate of growth of per capita production may be written as follows:

$$
\frac{1}{Y(t)} \frac{d Y(t)}{d t}=\frac{\delta(1+\gamma-\beta)-\rho}{\beta-\gamma}+\frac{\delta \beta(1+\gamma-\beta)-\gamma \rho}{(1-\beta)(\beta-\gamma)}\left[\frac{(\bar{K})(t)}{\left(\frac{K}{Q}\right)(t)}\right]^{1-\beta}
$$

Moreover, from Propositions 3 and 4, the rate of growth of physical capital stock, which is equal to the rate of growth of per capita consumption, may be written as follows:

$$
\frac{1}{K(t)} \frac{d K(t)}{d t}=\frac{1}{c(t)} \frac{d c(t)}{d t}=-\frac{\rho}{\beta}+\frac{\delta \beta(1+\gamma-\beta)-\gamma \rho}{(1-\beta) \beta(\beta-\gamma)}\left[\frac{(\bar{K})(t)}{\left(\frac{K}{Q}\right)(t)}\right]^{1-\beta}
$$

Now, it is easy to see that the previous rates of growth for $Y, c$ and $K$ are above or below their common long-run growth rate, $\bar{g}=\frac{1+\gamma-\beta}{1-\beta}\left(\frac{\delta-\rho}{\beta-\gamma}\right)$, depending on whether the ratio $\left(\frac{K}{Q}\right)$ is below or above its long-run level $(\bar{K})$. The latter, in turn, depends on whether $Q_{0} \gtreqless\left(\frac{\delta \beta(1+\gamma-\beta)-\gamma \rho}{(\beta-\gamma) \beta C_{0}^{0}}\right)^{\frac{1}{1+\gamma-\beta}}\left(\frac{\rho}{\beta}\right)^{\frac{1-\beta}{1+\gamma-\beta}}$ $K_{0}^{\frac{1-\beta}{1+\gamma-\beta}}$. Hence, we may conclude that transitional rates of growth converge, from above or below, to their long-run values. This convergence is a direct consequence of the convergence to unity experienced by the ratio: 


$$
\begin{gathered}
\left(\frac{\left(\frac{K}{Q}\right)(t)}{\left(\frac{K}{Q}\right)(t)}\right)^{1-\beta}= \\
1+\left[\frac{\left(\frac{\rho}{\beta} K_{0}\right)^{1-\beta}(\delta \beta(1+\gamma-\beta)-\gamma \rho)}{\left.(\beta-\gamma) \beta C_{0}^{0} Q_{0}^{1+\gamma-\beta}-1\right] \exp \left\{-\frac{\delta \beta(1+\gamma-\beta)-\gamma \rho}{\beta(\beta-\gamma)} t\right\}}\right.
\end{gathered}
$$

The previous result holds in both situations: positive long-run rates of growth when $\delta>\rho$, and negative long-run rates of growth when $\delta<\rho$. In the former, convergence from above occurs for short-run rates of growth always positive, but convergence from below may be found even for initially negative shortrun rates of growth crossing the zero axis in finite time. In the second one, convergence from below occurs for short-run rates of growth always negative, but convergence from above may be found even for initially positive short-run rates of growth crossing the zero axis in finite time.

Coming back to the levels of the variables, and according to the Propositions provided in the previous section, we may conclude that in the short-run trajectories for $K, c$ and $Y$ show transitional dynamics as well as convergence to their long-run levels and rates of growth. The trajectory for the shadow price of physical capital stock, $\theta_{1}$, also shows transitional dynamics and convergence in its level and rate of growth. Moreover, we find that price and quantity evolve in opposite directions. On the other hand, we prove that there are no transitional dynamics for $Q$ and $z$. This is also true for $\theta_{2}$, the shadow price of natural capital stock, which along the unique equilibrium trajectory evolves exponentially at a constant rate. In this case price and quantity move in opposite directions too. As a consequence of the previous statements, the trajectories for relative prices and the ratio between capital stocks experience transitional dynamics and convergence in both their levels and rates of growth.

It has to be remarked that, although the long-run trajectory for $Q$ depends on the initial condition $Q_{0}$, the long-run trajectories for $K$ and $Y$ do not depend on the initial condition $K_{0}$, but only on the initial condition $Q_{0}$. This means that what matters for the long-run levels of (per capita) production is 
not the initial endowment of physical capital but the endowment of natural capital. ${ }^{13}$ The best way to see this is by taking two similar countries A and $\mathrm{B}$, and allowing differences among them only in their initial endowments of physical and natural capital. It is beyond question that they will converge to the same rate of growth unless some fundamental difference affects their technological or preference parameters. However, during the transition their growth rates may differ due to differences in the levels of both natural and physical capital stocks. According to (26), the current rate of growth of (per capita) production depends positively on the distance between the current level and the level associated with the balanced growth path of the ratio between physical and natural capital stocks. But things are different in terms of the levels of output (per capita), since there could be overtaking, divergence without overtaking or convergence, depending on the initial conditions. ${ }^{14}$ First, consider a resource-poor country A relative to another resource-rich country B, $Q_{0}^{A}<Q_{0}^{B}$, but the former being endowed with a bigger initial physical capital stock, $K_{0}^{A}>K_{0}^{B}$. Then, as long as the initial current production in country $\mathrm{A}$ is still bigger than in country $\mathrm{B}, Y^{A}(0)>Y^{B}(0)$, this one will overtake country $\mathrm{A}$ in a finite time period given that in the long-run we will observe $\bar{Y}^{A}<\bar{Y}^{B}$. Here, overtaking appears as absolutely dependent on the initial endowments of natural capital across countries and does not depend on the rate of extraction that they choose during the transition. Consequently, the resource-rich country will always emerge in finite time as the richest country. Second, pure divergence without overtaking also appears in this model when we observe a relatively resource-rich country A, $Q_{0}^{A}>Q_{0}^{B}$, endowed with a higher initial physical capital stock, $K_{0}^{A} \geqq K_{0}^{B}$. In such a case we have $Y^{A}(0)>Y^{B}(0)$ and also that $\bar{Y}^{A}>\bar{Y}^{B}$ in the long-run. Third, we can still observe convergence in levels for any initial distribution of physical capital stocks, $K_{0}^{A} \gtrless K_{0}^{B}$, if we have two equal resource-rich countries, $Q_{0}^{A}=Q_{0}^{B}$. In such a case, although $Y^{A}(0) \gtrless Y^{B}(0)$, we will observe that in the long-run $\bar{Y}^{A}=\bar{Y}^{B}$ always holds. In short, these cases show how much

\footnotetext{
${ }^{13}$ This particular result arises from the fact that we have assumed the inverse of the intertemporal elasticity of substitution to be equal to the physical capital share. However, although in a more general framework the model will not display exactly the same kind of results, this one may still be considered as an orientative case for the study of more realistic and complex growth processes.

${ }^{14}$ This is true only for the case where variables experience positive growth because in the opposite case, where $\delta<\rho$, the only feasible result is convergence, but convergence to zero.
} 
relevant is natural capital over physical capital, in determining the pattern of growth followed by different countries over time.

Next, we will provide some analytical expressions to easily handle the results on convergence studied along the previous paragraphs. First, taking the definition of speed of convergence and using (20), (21), (23) and (25), we deduce the following result:

$$
S C(t) \equiv \frac{-\frac{d}{d t}\left[\ln \left(\frac{Y(t)}{\bar{Y}(t)}\right)\right]}{\ln Y(t)-\ln \bar{Y}(t)}=\frac{\delta \beta(1+\gamma-\beta)-\gamma \rho}{(\beta-\gamma) \beta}\left[\frac{(\bar{K})(t)}{\left(\frac{K}{Q}\right)(t)}\right]^{1-\beta}
$$

This equation gives the speed of convergence of the current production level to the long-run production level. The speed of convergence approaches, from above or below, to the long-run value $\lim _{t \rightarrow \infty} S C(t)=\frac{\delta \beta(1+\gamma-\beta)-\gamma \rho}{(\beta-\gamma) \beta}>0$, depending on whether $\frac{\left(\frac{K}{Q}\right)(t)}{\left(\frac{\bar{K}}{Q}\right)(t)} \lesseqgtr 1$. The long-run value of the speed of convergence depends positively on the natural resource intrinsic growth rate, $\delta$, and negatively on the intertemporal rate of discount, $\rho$. Moreover, it depends positively on the parameter associated with technological externality, $\gamma$, when $\delta>\rho$ but negatively when $\delta<\rho$. The effect of the elasticity of output with respect to physical capital, $\beta$, remains ambiguous due to the presence of the externality.

In general, to the extent that in the context of our model as countries develop their current $\frac{K}{Q}$ values, and hence also their levels of per capita production, approach the long-run ones from below, then (29) shows that the speed of convergence decreases during the transition. Furthermore, as long as sustainability is ensured, the rates of growth during the transition are always positive. The picture that Rodríguez and Sachs (1999) describe in terms of short-run evolution for per capita production has nothing to do with ours. They find for a developing country that convergence to the steady state levels occurs from above and, associated with the nature of their model, also predict negative rates of growth during the transition. According to these authors, natural capital abundance allows an economy to afford extraordinary consumption possibilities and, hence, on the transition to the steady state it grows more slowly precisely because they have an unsustainable high level of income. Nevertheless, the process of convergence in our model is 
monotonic and, consequently, it does not produce anything comparable to their mechanism of overshooting.

Finally, from the definition of saving rate that corresponds to this model economy, and using expressions for $K, c$ and $Y$ as well as (23) and (25), we deduce the following result:

$$
s(t) \equiv 1-\frac{c(t)}{Y(t)}=1-\frac{\rho}{\beta} \frac{K(t)}{Y(t)}=1-\frac{(1-\beta) \rho(\beta-\gamma)}{\delta \beta(1+\gamma-\beta)-\gamma \rho}\left[\frac{\left(\frac{K}{Q}\right)(t)}{(\bar{K})(t)}\right]^{1-\beta}
$$

Under the condition which guarantees positive economic growth and sustainability: $\delta>\rho$, the saving rate converges, from above or below, to its unique long-run value $0<\lim _{t \rightarrow \infty} s(t)=\frac{(1+\gamma-\beta) \beta(\delta-\rho)}{\delta \beta(1+\gamma-\beta)-\gamma \rho}<1$, depending on whether $\frac{\left(\frac{K}{Q}\right)(t)}{\left(\frac{\bar{K}}{Q}\right)(t)} \lesseqgtr 1$. This long-run saving rate depends positively on $\delta$, as well as on the size of the externality parameter, $\gamma$. Moreover, it depends negatively on the discount rate, $\rho$, but the sign of the effect associated with $\beta$, the aggregate physical capital share, is ambiguous. This sign will be positive or negative depending on whether $\beta \gtrless \frac{1+\gamma}{2}$, if and only if $\gamma \neq 0$. Otherwise, this latter parameter would not have any influence on the asymptotic saving rate.

The result given in (30) allows us to connect with the well-known Hartwick's Rule that has been largely studied in the context of neoclassical growth models when a non-renewable natural resource, which is subject to depletion, is included. This is a savings rule that concerns the rate at which physical capital must be accumulated if we want to compensate for the diminishing natural resource. ${ }^{15}$ The Hartwick rule ensures that an aggregate measure of capital is being maintained at a constant level, but also imposes strong conditions like efficient extraction and substitutability between natural and physical capital. Then, by following this rule we increase the feasibility of having a sustained level of per capita consumption in the future. Notwithstanding, what we have found in our model is a general savings rule, which determines the amount of physical capital that has to be accumulated in order to justify

\footnotetext{
${ }^{15}$ Rodríguez and Sachs (1999) suggest that an economy has to invest its temporary resource windfalls in international assets that pay permanent annuities, and consume the interest it earns on such assets.
} 
a positive long-run rate of growth of per capita consumption. Furthermore, this rule is obtained along with conditions that ensure an increasing stock of natural capital as well as an efficient harvesting programme. ${ }^{16}$

In a recent paper, Gylfason and Zoega (2001) analyze from a theoretical point of view the influence of natural resource abundance on the saving rate, the rate of growth and the speed of convergence. They carry out the above study using both a neoclassical growth model as well as an endogenous growth model à la Romer, expanded to include natural resources. In the context of the former, authors show that the share of natural capital in national income has a negative impact on the saving rate as well as on the long-run level of output per capita, but a positive one on the speed of convergence. In the context of the second model, they show that either the saving rate as well as the long-run rate of growth depend negatively on the natural capital share. For now, in a two-sector endogenous growth model, our results show that the long-run value of the saving rate does not depend on the share of natural capital in national income, $1-\beta$, if we make comparable the models assuming $\gamma=0$. We also confirm their result concerning the speed of convergence but, contrary to them, we find that the long-run rate of growth depends positively on the natural capital share.

\section{Conclusions}

Our results show that it is possible to have simultaneously economic growth and sustainability, compatible with efficient behavior in both senses: intra and inter-temporally. In economies with impatient agents, however, positive long-run growth is only possible if their natural resource experiences a high biotic rate of growth. In the short-run production and consumption show transitional dynamics as well as convergence to their long-run levels and

\footnotetext{
${ }^{16}$ Actually, our model admits both a positive as well as a negative long-run rate of growth, which is determined by the relationship between the efficient harvesting rate, $\bar{z}$, and the critical value $z^{S}$. That is to say, the relationship between $\delta$ and $\rho$. So, given a low harvesting rate that implies an increasing stock of natural capital, the only opportunity for balanced long-run positive growth comes from a simultaneous increase of the physical capital stock. A positive saving rate, and the corresponding rate of physical capital accumulation, is required to ensure such a result. In the alternative case where a high harvesting rate determines a decreasing stock of natural capital, our model allows for a negative saving rate that implies a decreasing stock of physical capital.
} 
rates of growth. In general, any increase in the intrinsic rate of growth of the natural resource expands the margins for sustainability and has an additional positive effect on the long-run rate of growth. Moreover, a more patient society chooses a lower harvesting rate and reaches a greater longrun rate of growth. In short, it has to be noted the prominent importance of natural capital for growth. In the particular case of no externality affecting production in the final goods sector, our model predicts that the saving rate does not depend on the natural capital share, but the long-run rate of growth and the speed of convergence depend positively, while the extraction rate depends negatively. Thus, in the context of our model, we can explain most of the empirical facts described in Gylfason and Zoega (2001) and Gylfason (2001a, 2001b). If we consider two differently endowed economies, they will converge in rates of growth whenever they have identical parameters associated with production and utility functions. But things are different in terms of the levels of the variables, since there could be overtaking, divergence without overtaking or convergence, depending on the initial conditions.

All the previous results have been obtained under the simplifying assumption that the inverse of the intertemporal elasticity of substitution equals the physical capital share. Nevertheless, given that we are interested in theoretical properties of the transitional dynamics and the closed form solution for the different variables, the above assumption does not seem too restrictive. In fact, we can identify three main shortcomings associated with this procedure: consumption is proportional to physical capital stock, the initial physical capital stock does not appear determining any of the long-run balanced growth paths, and transitional dynamics for the variables in the model are partially simplified. Consequently, our model may be considered as an orientative theory for the study of growth processes in economies where production is obtained using a renewable natural resource in combination with other factors.

\section{References}

[1] Auty, R. M. (2001) The Political Economy of Resource-Driven Growth. European Economic Review 45, 839-846.

[2] Aznar-Márquez, J. and J. R. Ruiz-Tamarit (2002) Closed-Form Solution for a Two-Sector Endogenous Growth Model with two Controls. Mimeo, 
Universitat de València (Spain).

[3] Benhabib, J. and R. Perli (1994) Uniqueness and Indeterminacy: On the Dynamics of Endogenous Growth. Journal of Economic Theory 63, 113-142.

[4] Gylfason, T. (2001a) Natural Resources, Education and Economic Development. European Economic Review 45, 847-859.

[5] Gylfason, T. (2001b) Nature, Power and Growth. Scottish Journal of Political Economy 5, November.

[6] Gylfason, T., T. T. Herbertsson and G. Zoega (1999) A Mixed Blessing: Natural Resources and Economic Growth. Macroeconomic Dynamics 3, 204-225.

[7] Gylfason, T. and G. Zoega (2001) Natural Resources and Economic Growth: The Role of Investment. CEPR Discussion Paper No 2743, March.

[8] Lucas, R. E., Jr. (1988) On the Mechanics of Economic Development. Journal of Monetary Economics 22, 3-42.

[9] Perman, R., Y. Ma, J. Mcgilvray and M. Common (1999) Natural Resource and Environmental Economics. England: Pearson Education Limited.

[10] Peterson, F. M. and A. C. Fisher (1977) The Exploitation of Renewable and Nonrenewable Natural Resources. Economic Journal 87, 681-721.

[11] Rodríguez, F. and J. D. Sachs (1999) Why Do Resource-Abundant Economies Grow More Slowly?. Journal of Economic Growth 4, 277303.

[12] Ruiz-Tamarit, J. R. (2002) Multiplicity, Overtaking and Convergence in the Lucas Two-Sector Growth Model. FEDEA, Documento de Trabajo 2002-17.

[13] Sachs, J. D. and A. M. Warner (1995) Natural Resource Abundance and Economic Growth. NBER, Working Paper No. 5398. 
[14] Sachs, J. D. and A. M. Warner (2001) The Curse of Natural Resources. European Economic Review 45, 827-838.

[15] Shone, R. (1997) Economic Dynamics: Phase Diagrams and their Economic Application, New York: Cambridge University Press.

[16] Stiglitz, J. E. (1980) A Neoclassical Analysis of the Economics of Natural Resources. NBER (Reprints), Working Paper No. 0077. 